\title{
Fast Screening in Large Face Databases Using Merit-Based Dominant Points
}

\author{
Yongsheng Gao \\ School of Microelectronic Engineering, Griffith University, Australia, \\ yongsheng.gao@griffith.edu.au
}

\begin{abstract}
Current face identification approaches require computer systems to search through large quantity of face feature sets in the database and pick the ones that best match the features of an unknown input face. In this paper, a fast screening method for large face database searching is proposed. The method utilizes dominant points instead of edge maps as features for similarity measurement. A new formulation of Hausdorff distance is designed for merit-based dominant point matching. The screening experiments demonstrated that the proposed face screening method significantly improves the computational speed and the storage economy. It provides a very efficient way for large face databases searching and screening.
\end{abstract}

\section{Introduction}

Computerized human face recognition has been an active research area for recent years. It has many practical applications, such as bankcard identification, mug shots searching, access control, security monitoring and surveillance systems. Face recognition is used to identify one or more persons from still images or a video image sequence of a scene by comparing input images with faces stored in a database. In a face identification system, face features are extracted/coded off-line from the original images, and stored in the face feature database. In the identification stage, the same features are extracted from the input face, and the features of the input image are compared with the features of each model images in the database. In most systems, searching is the most computationally expensive operation due to the large number of images available in the database. Fast screening algorithms are prerequisite of identification systems. Takács [1] proposed a face matching and fast screening approach for large face databases. The similarity measure is conducted using a modified Hausdorff distance on edge maps of face images. 92\% accuracy was achieved in their identification experiment. He argued that the process of face recognition might start at a much earlier stage and edge images could be used for fast screening of faces without the involvement of high-level cognitive functions.

As a fast screening technique, the computational efficiency is an important issue. This study proposes an efficient method for fast screening in large face databases using merit-based dominant points. It significantly reduces the computational complexity and the storage requirement. Experimental results demonstrate that the proposed approach is 28 times faster than that of the method in [1] with a cost of $1.74 \%$ accuracy degradation. Furthermore, the storage space is reduced to $18.5 \%$ of the existing method.

In Section 2, the dominant point detection together with a brief discussion on edge detection is presented. In Section 3, a new formulation of Hausdorff distance based on merit for dominant point matching is proposed. Encouraging experimental results are reported in Section 4. Finally, Section 5 contains concluding remarks.

\section{2. dominant point detection}

Edges are the most fundamental features of objects in the $3 \mathrm{D}$ world. The edges in an image reflect large local intensity changes that are caused by the geometrical structure of the object, the characteristics of surface reflectance of the object, and the viewing direction. Edge detection is an important technique in computer vision and pattern recognition systems since the edges usually correspond to some important properties of objects, such as object shape and boundary.

Edge extraction has been studied most extensively, and many reliable algorithms have been proposed and implemented. [2-6] are only a few examples from a large collection of algorithms that have been presented to the computer vision community. The continuing development of edge detectors is producing increasingly complex edge detection algorithms. However, the "increased sophistication is not producing a commensurate improvement in 
performance" [7]. M. D. Heath et al. [8, 9] had investigated the performance of different edge detectors. They compared the edge detectors based on experimental psychology and statistics, in which humans rated the outputs of low-level vision algorithms. One of their clear results was that "no one single edge detector was best overall; for any given image it is difficult to predict which edge detector will be best" [9].

The proposed face matching method using dominant points as features does not rely on any specific edge detector. It is a general method that any edge detector can be used. In this study, an edge detector based on the algorithm of Nevatia [6] is used followed by a thinning process to generate one pixel wide edge curves. The dynamic two-strip algorithm (Dyn2S) [10] is utilized to detect dominant points, the points with high curvatures, on the facial edge curves. In the Dyn2S algorithm, a strip is fitted to the left and right of each point on the curve, and the points inside each strip are approximated as a straight line. The orientation and width of the strip are adjusted automatically. Longer and narrower strips are favored. The curvature of each point is computed as the angle subtended by its two strips. Then a measure of "merit" based on the strips' lengths and widths, and the angle between the strips is calculated. A local maximum detection process is applied to select points of high curvature. This merit provides an objective evaluation of the prominent strength of each point and will be used as a significance measure of the dominant point in the proposed modified Hausdorff distance measure. The results of applying these processes on human faces are illustrated in Figure 1.

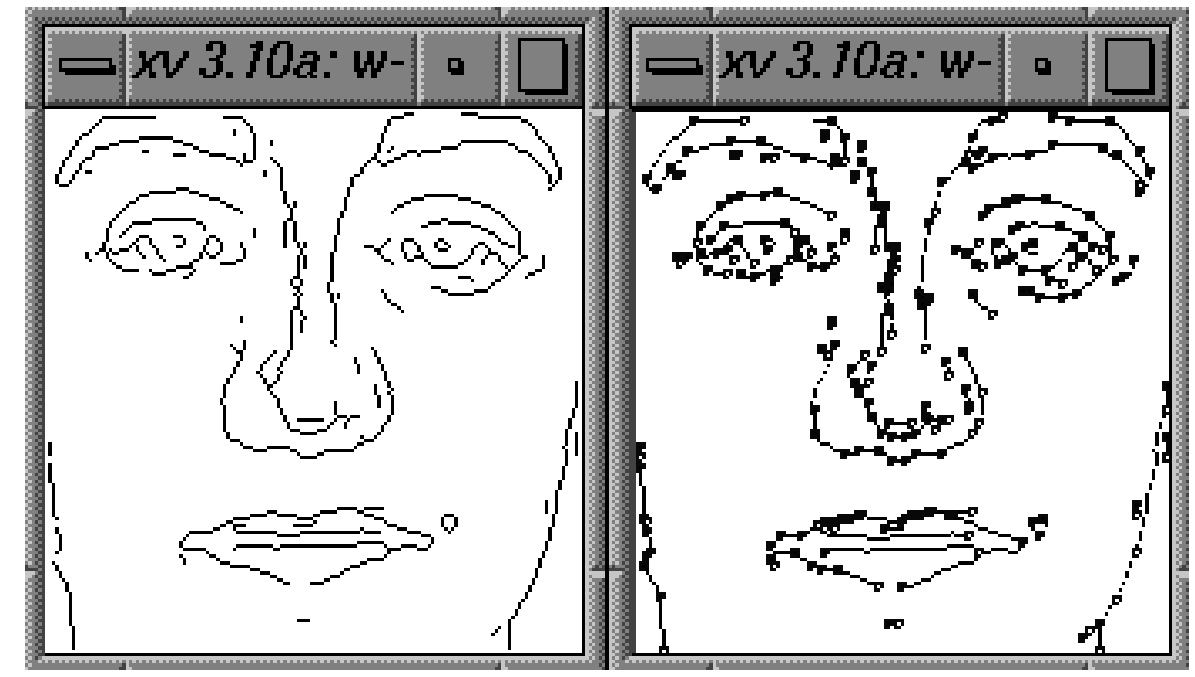

Figure 1. An illustration of a face edge map (left) and the dominant points superimposed on the edge map (right).

\section{Merit-based Hausdorff distance}

The Hausdorff distance is a shape comparison metric based on binary images. It is a distance defined between two point sets. Unlike most shape comparison methods that build a point-to-point correspondence between a model and a test image, Hausdorff distance can be calculated without explicit point correspondence. Huttenlocher et al. [11] argued that the Hausdorff distance for binary image matching is more tolerant to perturbations in the locations of points than binary correlation techniques since it measures proximity rather than exact superposition. Here, we propose a new merit-based Hausdorff distance
(MBHD) measure for dominant point matching instead of binary image matching.

Given two finite point sets $M=\left\{m_{1}, m_{2}, \ldots\right.$, $\left.m_{p}\right\}$ (representing a model in the database) and $T=$ $\left\{t_{1}, t_{2}, \ldots, t_{q}\right\}$ (representing a test image), the Hausdorff distance is defined as

$$
H(M, T)=\max (h(M, T), h(T, M)),
$$

where

$$
h(M, T)=\max _{m_{i} \in M t_{j} \in T}\left\|m_{i}-t_{j}\right\|,
$$

and $\left\|m_{i}-t_{j}\right\|$ is the Euclidean norm of the points 
$m_{i}$ and $t_{j}$. The function $h(M, T)$ is called the directed Hausdorff distance from $M$ to $T$. It identifies the point $m_{i} \in M$ that is the farthest from any point of $T$ and measures the distance from $m_{i}$ to its nearest neighbor in $T$. The Hausdorff distance $H(M, T)$ is the maximum of $h(M, T)$ and $h(T, M)$. Thus, it measures the degree of mismatch between two sets by measuring the distance of the point of $M$ that is farthest from any point of $T$, and vise versa.

Dubuisson and Jain [12] investigated 24 forms of different Hausdorff distance measures and indicated that a Modified Hausdorff Distance (MHD) measure has the best performance. The directed MHD is defined as

$$
h(M, T)=\frac{1}{P} \sum_{m_{i} \in M} \min _{t_{j} \in T}\left\|m_{i}-t_{j}\right\|,
$$

where $P$ is the number of points in $M$. The definition of the undirected MHD is the same as (1). The Hausdorff distance defined as (1) and (2) is very sensitive to outlier points. A few outlier points, even only a single one, can perturb the distance greatly, though the two objects might be very similar. The MHD can alleviate the sensitivity of the Hausdorff distance, which uses equation (1), to outlier points.

In this paper, MBHD is employed to match dominant points rather than binary images. Dominant point representation demands much less storage requirement and thus is more computationally efficient than binary images. The merit value of each dominant point is taken into the computation of the distance. The directed MBHD is defined as

$$
h_{M B H D}(M, T)=\frac{1}{\sum_{m_{i} \in M} W_{m_{i} t_{j}}} \sum_{m_{i} \in M} W_{m_{i} t_{j}} \cdot \min \left\|m_{t_{j} \in T}-t_{j}\right\| \text {, }
$$

where $W_{m_{i} t_{j}}=\frac{1}{2}\left(W_{m_{i}}+W_{t_{j}}\right)$ is the average merit of dominant points $m_{i}$ and $t_{j}$. In this study, $W_{m_{i}}$ and $W_{t_{j}}$ are merits provided by the Dyn2S algorithm previously mentioned. Other dominant point detection algorithms, which provide merits or significances of points, can also be used. Every $\min _{t_{j} \in T}\left\|m_{i}-t_{j}\right\|$ (i.e., the distance of a matched pair) is weighted by the average merit of $m_{i}$ and $t_{j}$ because its contribution to

$h_{M B H D}(M, T)$ is assumed to be propotional to the significances of the two dominant points. $h_{M B H D}(M, T)$ identifies its nearest neighbor point in $T$ and attaches a merit-based weight to the distance from $m_{i}$ to the identified point. The undirected MBHD is defined as follow.

$$
H_{M B H D}(M, T)=\max \left(h_{M B H D}(M, T), h_{M B H D}(T, M)\right)
$$

\section{Experimental results}

Two public available face databases were used in our fast screening experiments. The first face database [13] is from the University of Bern. It contains of 30 persons with 2 frontal and 2 profile views per person. Each image has the size of $512 \times 342$ pixels with 256 gray levels and variations of the head position, size, and contrast. The lighting conditions during image acquisition were carefully controlled. One profile and one frontal face images of each person were used as models while the other pair was used as input in the frontal and the profile face matching experiments, respectively. Though there are 60 pairs of models and inputs, one can have 120 matching tests if the roles of model and input are interchanged. Sample sets of the frontal and the profile faces are shown in Figures 2 and 3.

In order to remove the influences of hair, neck, and other foreign objects such as shirt collars, the face matching process should be restricted to the personal identity area containing only the eyebrows, eyes, nose, mouth, and chin. For this purpose, the automatic profile location algorithm [15] was employed to detect the nose tip and chin points. The distance between these two points was used as a reference to normalize the image size, align the face position, and crop the facial area. For the frontal faces, the eye locations are detected for normalization, alignment, and cropping. 


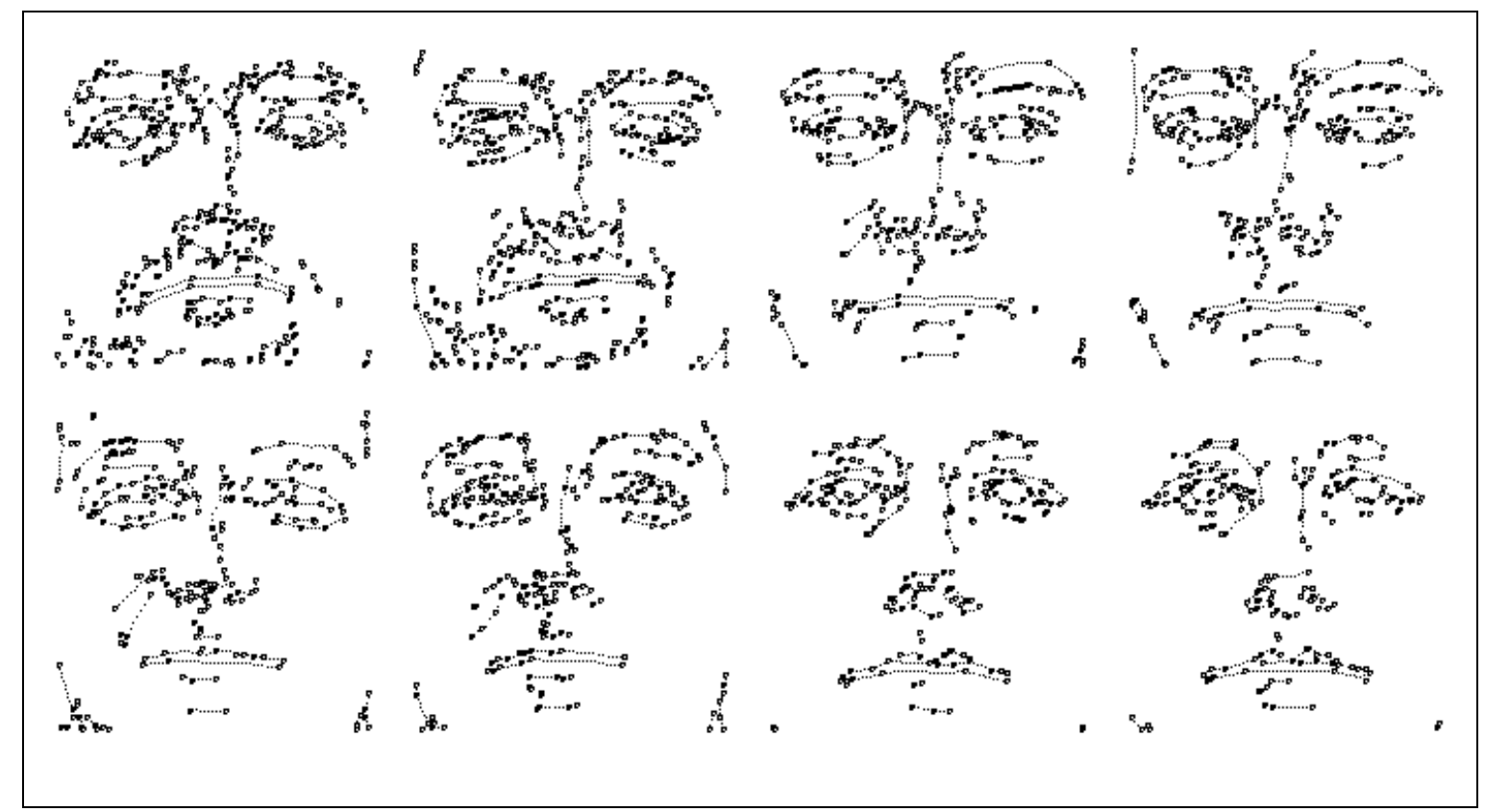

Figure 2. Sample pairs of face edge map with detected dominant points

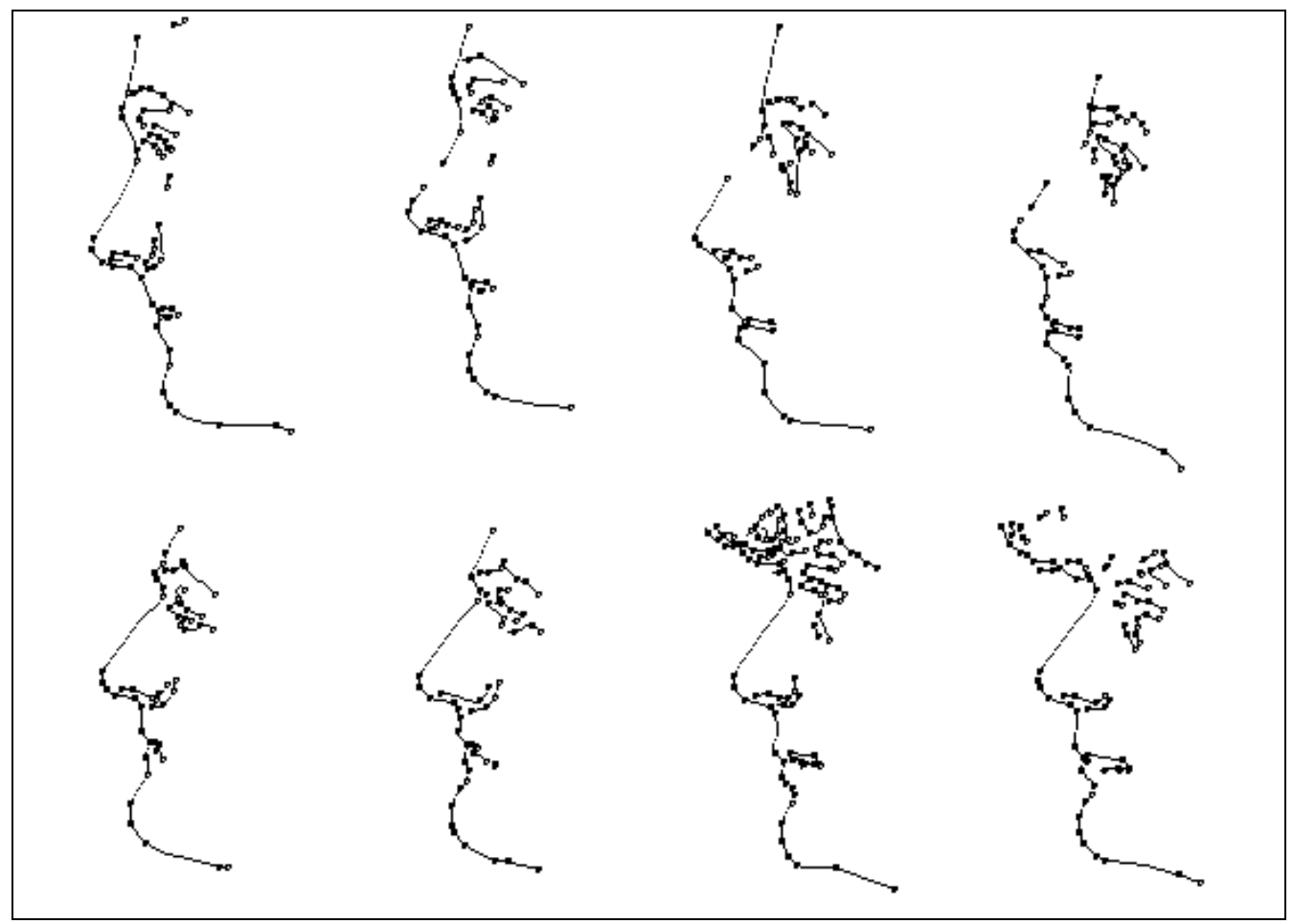

Figure 3. Sample pairs of profile edge map with detected dominant points.

The identification accuracy using the existing edge image (EI) method and the proposed dominant point (DP) method are tabulated in Table 1. Their storage sizes are summarized in Table 2 . The proposed meritbased dominant point matching correctly identified
$94.17 \%$ of the testing faces. This rate only dropped $1.74 \%$ from the $95.84 \%$ of the EI method. However, its average storage space significantly dropped $81.5 \%$. The computational complexities of MHD and MBHD are both of the order $O(P Q)$ for point sets of size $P$ and 
$Q$. In other words, the computational time of MBHD is only $3.4 \%\left(=(18.5 \%)^{2}\right)$ of that of MHD. These results show that compared to the MHD method, the MBHD method only required $3.4 \%$ of the computational time and $18.5 \%$ of the storage space. The cost to the above benefits was only a $1.74 \%$ decrease in accuracy. Computing efficiently the MHD and the $\mathrm{M}^{2} \mathrm{HD}$ can be done by preprocessing the point sets in time $O(P \log P)$ and $O(Q \log Q)$ into a data structure requiring $O(P)$ and $O(Q)$ space (e.g., a Voronoi diagram in the plane), and then running queries in time $O(\log P)$ and $O(\log Q)$ per query. (See [16-18] for technical details.) Thus, the desired measures can be computed in time $O((P+Q) \log (\max (P, Q)))$.

The second face database is from Purdue University [14]. For the details on the collection of the database, readers can refer to [19]. 224 images of 112 subjects were tested. The 112 images of 112 people taken in the first session were used as models while the other 112 images taken after two weeks were used as testing images. Note that this is a single model test.

Table 1 The accuracy (\%) comparison of edge image (EI) and dominant point (DP).

\begin{tabular}{|c|c|c|c|}
\hline & EI & DP & Decrease (\%) \\
\hline Accuracy & 95.84 & 94.17 & $1.74 \%$ \\
\hline
\end{tabular}

Table 2 Comparison of average storage space (\# of points).

\begin{tabular}{|c|c|c|c|c|}
\hline & EI & DP & $\frac{\text { Dominant points }}{\text { Edge map }}$ & $\begin{array}{c}\text { Decrease } \\
(\%)\end{array}$ \\
\hline Frontal view & 1451 & 270 & $18.6 \%$ & $81.4 \%$ \\
\hline Profile view & 423 & 78 & $18.4 \%$ & $81.6 \%$ \\
\hline Average & - & - & $18.5 \%$ & $81.5 \%$ \\
\hline
\end{tabular}

The experimental results are summarized in Figure 4 in terms of top $\mathrm{N}$ screening/identification. In the top 1 identification, the correct match is only counted when the best matched face from models is the identical face (i.e., the face image from the same person) of the input. In the top $\mathrm{N}$ screening, the correct match is counted when the identical face of the input is among the best $N$ matched faces from models. It was found that the accuracy of the proposed DP matching is slightly lower than that of the EI matching (see Figure 4). The DP approach correctly identified all the testing images using top 13 screening (i.e., screening the best $13 / 112=11.6 \%$ matches), while EI method correctly identified them using top 10 screening (i.e., screening the best $8.9 \%$ matches). The screening result of the DP approach, which could be used as candidates of another fine tuning matching process, increased by $2.7 \%$. On the other hand, the computational time and the storage space of the DP approach were reduced to $3.7 \%$ $\left(=(19.3 \%)^{2}\right)$ and $19.3 \%$ of those of EI, respectively (see Table 3). The MHD matching applied to dominant points was also tested and plotted in Figure 4 as a comparison reference. Note that the top 24 screening (i.e., screening the best $21.4 \%$ matches) is required by the MHD matching on dominant points to achieve $100 \%$ accuracy.

Table 3 Comparison of average storage space (\# of points).

\begin{tabular}{|c|c|c|c|c|}
\hline & Edge image & Dominant point & $\frac{\text { Dominant points }}{\text { Edge map }}(\%)$ & $\begin{array}{c}\text { Decrease } \\
(\%)\end{array}$ \\
\hline Storage space & 1401 & 271 & $19.3 \%$ & $80.7 \%$ \\
\hline
\end{tabular}




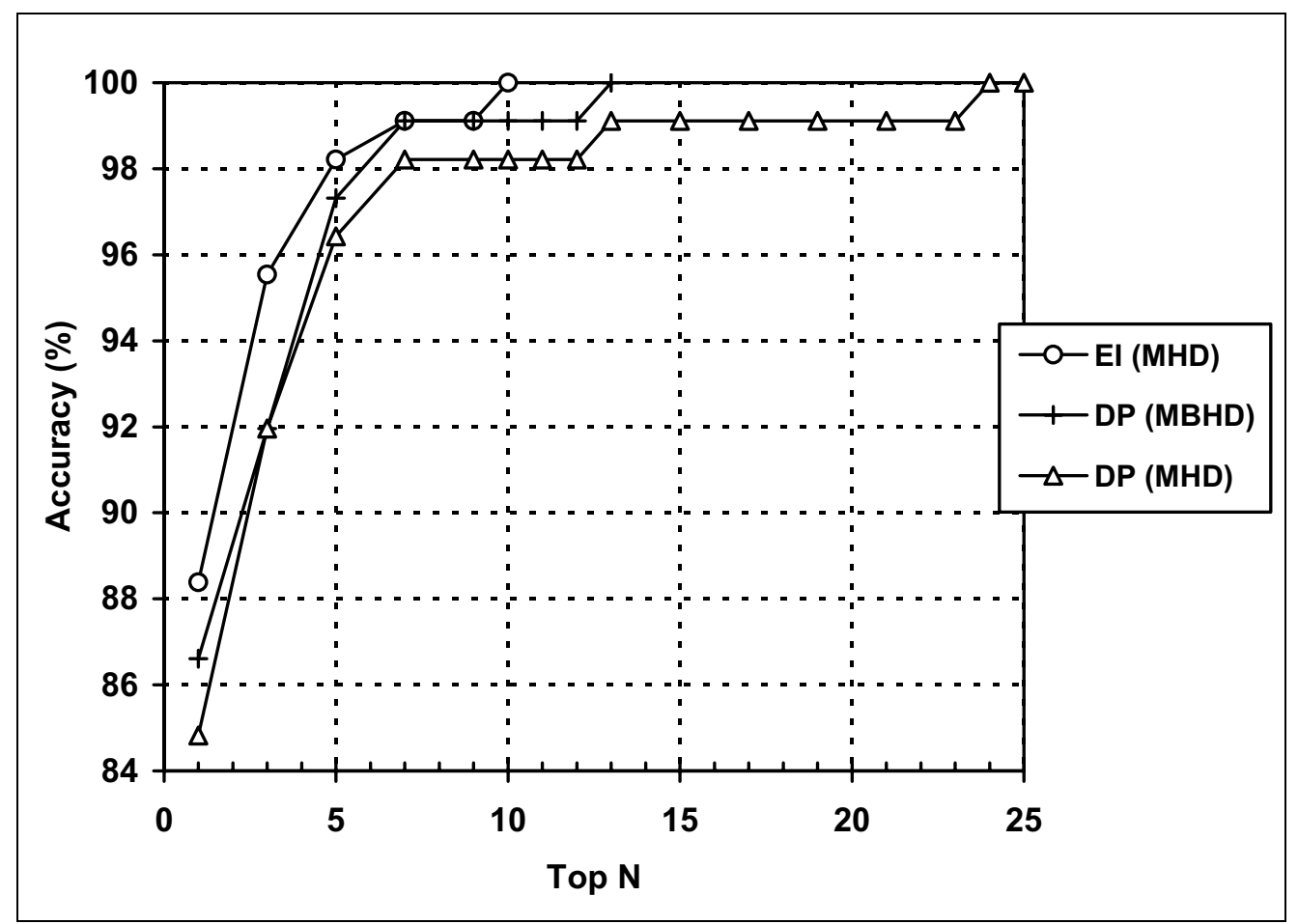

Figure 4. Experimental results of fast screening. El: edge image. DP: dominant point.

\section{Conclusion}

Current face identification approaches require computer systems to search through large quantity of face feature sets in the database and pick the ones that best match the features of an unknown input face. In this paper, a fast screening method for large face database searching is proposed. The method utilizes dominant points instead of edge maps as features for similarity measurement. A new formulation of Hausdorff distance is designed for merit-based dominant point matching. The screening experiments demonstrated that compared to the existing edge image approach, the proposed merit-base dominant point approach only required $3.4 \%$ of the computational time and $18.5 \%$ of the storage space. The cost to the above benefits was only a $1.74 \%$ decrease in accuracy. These results indicate that the proposed face screening method significantly improves the computational speed and the storage economy. It provides a very efficient way for large face databases searching and screening.

\section{References}

[1] TAKÁCS, B.: 'Comparing Face Images Using the Modified Hausdorff Distance,' Pattern Recognition, 1998, 31, pp. 1873-1881
[2] MARR, D. and HILDRETH, E. C.: 'Theory of Edge Detection,' Proceedings Royal Society of London, 1980, pp. 187-217

[3] IVERSON, L. A. and ZUCKER, S. W.: 'Logical/Linear Operators for Image Curves,' IEEE Transactions on Patten Analysis and Machine Intelligence, 1995, 17, pp. 982-996

[4] SOBEL, I. E.: 'Cameral Model and Machine Perception,' PhD Thesis, Stanford University, 1970

[5] CANNY, J.: 'A Computational Approach to Edge Detection,' IEEE Transactions on Patten Analysis and Machine Intelligence, 1986, 8, pp. 679-698

[6] NEVATIA, R. and BABU, K. R.: 'Linear Feature Extraction and Description,' Computer Graphics and Image Processing, 1980, 13, pp. 257-269

[7] BOYER, K. L. and SARKAR, S.: 'Assessing the State of the Art in Edge Detection:1992,' SPIE, Application of Artificial Intelligence X: Machine Vision and Robotics, 1992, 1708, pp. 353-362

[8] HEATH, M. D., SARKAR, S., SANOCKI, T. and BOWYER, K. W.: 'A Robust Visual Method for Assessing the Relative Performance of Edge-Detection Algorithms,' IEEE Transactions on Patten Analysis and Machine Intelligence, 1997, 19, pp. 1338-1359

[9] HEATH, M. D., SARKAR, S., SANOCKI, T. and BOWYER, K. W.: 'Comparison of Edge Detectors: A Methodology and Initial Study,' Computer Vision and Image Understanding, 1998, 69, pp. 38-54

[10] LEUNG, M. K. H. and YANG, Y. H.: 'Dynamic TwoStrip Algorithm in Curve Fitting,' Pattern Recognition, 1990, 23, pp. 69-79 
[11] HUTTENLOCHER, D. P., KLANDERMAN, G. A. and RUCKLIDGE, W. J.: 'Comparing Images Using the Hausdorff Distance,' IEEE Transactions on Pattern Analysis and Machine Intelligence, 1993, 15, pp. 850863

[12] DUBUISSON, M. P. and JAIN, A. K.: 'A Modified Hausdorff Distance for Object Matching,' Proceedings $12^{\text {th }}$ International Conference on Pattern Recognition, 1994, Jerusalem, Israel, pp. 566-568

[13] Bern University face database. ftp://iamftp.unibe.ch/pub/Images/FaceImages/

[14] Purdue University face database. $<$ http://rvll.ecn.purdue.edu/ aleix/aleix face DB.html>

[15] GAO, Y.: 'Human Face Recognition Using Line Edge Information,' PhD Thesis, Nanyang Technological University, Singapore, 2000

[16] PREPARATA, F. P. and SHAMOS, M. I.: 'Computational Geometry-An Introduction,' New York: Springer-Verlag, 1985

[17] CHAZELLE, B. and EDELSBRUNNER, H.: 'An Improved Algorithm for Constructing kth-Order Voronoi Diagrams,' IEEE Transactions on Computers, 1987, C-36, pp. 1349-1354

[18] DE BERG, M., DEVILLERS, O., DOBRINDT, K. and SCHWARZKOPF, O.: 'Computing a Single Cell in the Overlay of Two Simple Polygons,' Information Processing Letters, 1997, 63, pp.215-219

[19] MARTINEZ, A. M. and BENAVENTE, R.: 'The AR Face Database,' CVC Technical Report \#24, June 1998 